\title{
Understanding and controlling the morphology of styrene-isoprene side-group liquid crystalline diblock copolymers
}

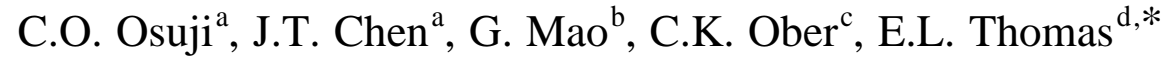 \\ ${ }^{\mathrm{a}}$ Tyco Electronics, Menlo Park, CA 94025, USA \\ ${ }^{\mathrm{b}} 3 \mathrm{M}$ Corporation, Austin, TX 78726, USA \\ ${ }^{c}$ Department of Materials Science and Engineering, Cornell University, Ithaca, NY 14850, USA \\ ${ }^{\mathrm{d}}$ Program in Polymer Science and Technology, Department of Materials Science and Engineering, Massachusetts Institute of Technology, Room 13-5094, \\ 77 Massachusetts Avenue, Cambridge, MA 02139, USA
}

Received 22 December 1999; received in revised form 9 March 2000; accepted 10 March 2000

\begin{abstract}
In side-chain liquid crystalline diblock copolymers, driving forces for ordering of the material may be provided by the chemical incompatibility between the blocks and by the liquid crystalline nature of one of the blocks. We study the microstructure and its development from initially isotropic solutions in side-group liquid crystalline copolymers based on styrene-isoprene diblocks. Hierarchical structure from the $5 \AA$ to the $500 \AA$ length scale is observed, the product of coherent block copolymer microphase separation and liquid crystalline mesophase formation. The presence of cylindrical microdomains of either the poly(isoprene-LC) or poly(styrene) block markedly increased the thermal stability of the LC. Confinement of the LC to cylindrical microdomains strongly inhibited defect formation within the mesophase after suitable orientation and thermal treatment, readily manifested by the significantly improved optical clarity of these samples versus LC matrix or LC lamellar samples. We consider the relative stabilities of parallel-transverse, perpendicular-parallel, parallel-parallel and transverse-perpendicular arrangements of the microdomain and mesophase structures in interpreting the development of structure in these materials under oscillatory shear. (C) 2000 Elsevier Science Ltd. All rights reserved.
\end{abstract}

Keywords: Diblock copolymers; Liquid crystalline nature; Side group

\section{Background}

Liquid crystalline block copolymers (LC BCPs) are a relatively new class of polymers, of commercial interest because of their broad range of applications, and of academic interest since they permit the study of structure formation under the influence of more than one driving force. The physics of their ordering is analogous to that in crystallizable block copolymers such as poly(ethylene- $b$ styrene) or poly(ethylene- $b$-3-methyl-1-butene) [1]. The formation of a liquid crystalline mesophase by one block typically requires the presence of a mobile mesogenic group in that block. Incorporation of liquid crystallinity into block copolymers is possible via various synthetic routes, and many different chain architectures such as segmented main-chain, rod-coil and side-group liquid crystalline block copolymers may be realized [2].

LC BCPs inherently possess a hierarchy of structure, with

\footnotetext{
* Corresponding author. Tel.: +1-617-253-6901; fax: +1-617-258-7874

E-mail address: elt@mit.edu (E.L. Thomas).
}

order present on the 5, 50 and $500 \AA$ A length scales, corresponding to the inter-mesogen, smectic layer and microdomain spacings, respectively. The manipulation of the chemistry and processing of liquid crystals or block copolymers to tailor their properties in some prescribed manner is a desirable goal. The driving forces for mesophase formation and microphase separation may also be similarly manipulated due to the tandem interaction of liquid crystallinity with microphase separation [3]. Interest in these materials is growing quickly, as their liquid crystallinity, coupled to their ability to self-assemble, makes them attractive for application to sensing and display devices based on their electro-optic, mechano-optic, and thermo-optic properties. Piezo-, pyro- and ferro-electric responses, for instance, can be elicited from these materials [4-8].

The concept of a liquid crystalline block copolymer was first fully realized by the synthesis by Percec and coworkers [9] of poly(para-vinyl benzene ether-b-biphenyl acrylate) and poly(para-vinyl benzene- $b-4-$ methoxy- 4 '-(ethoxy-(2ethoxy-(2-ethoxy))) biphenyl acrylate) based copolymers. Shortly thereafter, Adams and Gronski [10] produced a 


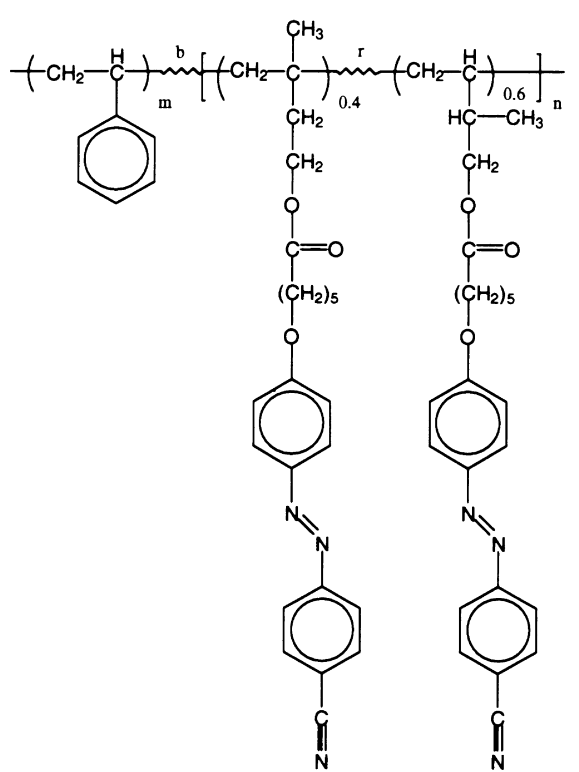

Fig. 1. Poly(stryrene- $b$-isoprene-LC) diblock copolymer.

styrene-butadiene diblock copolymer in which the butadiene block was side-functionalized with a cholesteryl mesogen. The aim of their synthesis was to prepare liquid crystalline phases of well defined shape and size so that the influence of the size of the phase on the LC behavior as well as the effect of the interface on the LC order in the boundary region could be investigated [11].

A systematic study of the influence of liquid crystallinity on microdomain structure and vice versa was conducted by Fischer et al. who produced a series of copolymers based on a cholesteryl functionalized ethyl methacrylate (PEMA) blocked first with styrene (PS) [12,13] then later with butyl methacrylate (PBMA)[14]. The homopolymer formed using the cholesteryl functionalized methyl methacrylate monomer displayed a smectic A structure. Using TEM (transmission electron microscopy) and SAXS (small angle X-ray scattering), in both types of copolymer systems they observed lamellae, spheres and cylinders of both PS and PBMA as well as spheres of the liquid crystalline block. Notably, the occurrence of cylinders of the LC component was not observed. In addition, a clear dependence of the

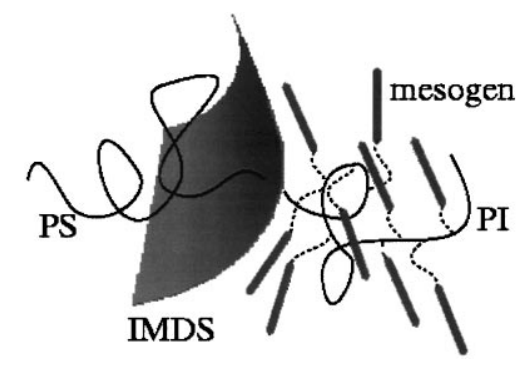

Fig. 2. Illustration of a section of the inter-material dividing surface, IMDS, for a cylindrical microdomain in a PS/PI-LC diblock copolymer. Each block occupies some area of the surface and is stretched away from it. The minority volume fraction PS block is on the concave side of the IMDS. mesophase type on the microphase separated structure was found. Specifically, the LC phase was nematic when confined to the discontinuous spherical domains, and smectic A otherwise. Clearly, the block copolymer morphology on the 100-1000 $\AA$ length scale was influencing the LC morphology on the smaller $10-40 \AA$ length scale. The anchoring condition of the mesogen with respect to the IMDS (inter-material dividing surface) was not determined, nor was the influence of spacer length explored. The noted absence of the cylindrical LC phase, as one might expect, is specific to the system and should not be universally expected $[2,15]$.

More recently, Hammond and coworkers [16,17] have synthesized a styrene-methyl methacrylate diblock copolymer in which the methyl methacrylate block is functionalized with a chiral mesogen using spacers of 6 and 10 carbon atoms. The mesogen in this case is based on a biphenyl benzoate core. Here coincidence is found between the order-disorder transition (ODT) and the LC isotropization temperature for block copolymers with high LC content and low molecular weight [7]. The situation is analogous to that of crystallizable block copolymers in the weak segregation limit in which microphase separation in driven by block crystallization. Currently, the synthesis of LC functionalized siloxane block copolymers is being pursued in an attempt to realize a smectic $\mathrm{C}^{*}$ phase at room temperature. This has been successfully accomplished for the siloxane LC as well as for a series of block copolymers [18].

Work conducted collaboratively by the Ober (Cornell) and Thomas (MIT) groups has focused on both LC rodamorphous coil block copolymers [19-23] and amorphous side-group LC-amorphous coil block copolymers $[6,15,19,20,24]$, as well as fluorinated side group LC copolymers [25]. The structure of the azobenzene mesogen based stryene-isoprene side-group LC diblock is shown in Fig. 1.

Using TEM and SAXS, microdomain structures of cylinders of poly(styrene), cylinders of poly(isoprene-LC) as well as lamellae were observed. Notably, cylinders were observed for $\Phi_{\text {PI-LC }}=0.91$, (volume fraction constituted by the isoprene backbone plus the attached LC mesogens) a volume fraction at which spheres are typically the most stable microdomain form in coil-coil diblock copolymers. The mesophase was smectic A in all cases for this series, and the mesogens were found, by X-ray diffraction from oriented samples, to anchor parallel to the IMDS, which is shown schematically in Fig. 2.

Dating from the original pioneering work of Keller et al. [26] in which a capillary rheometer was used to extrude and thus orient a cylinder forming styrene-butadiene-styrene triblock copolymer, various other external fields have been used to orient block copolymer microdomains. Oscillatory shear [27], electric fields [28] and roll-casting [29] have all been applied to produce various well aligned morphologies in di- and triblock copolymers. The presence of mechanical anisotropy on two different length scales due to two different but necessarily interacting sets of structures 
made for the interesting study of the morphology of the styrene-isoprene side group liquid crystalline materials under oscillatory shear. This manuscript is divided into two parts. The first, entitled Structure Formation, is a summary and critical discussion of the issues which may affect the morphology of these materials. Simple expressions are provided to describe the anchoring mediated free energy associated with the accommodation of an LC mesophase within or around the periodic IMDS of the block copolymer microdomains. In the second part, Experimental Results and Discussion, results are provided which reflect on the salient points of the first part. In particular, the effects of confinement on the characteristics of the LC mesophase and the effect of the mesophase on the orientation of the block copolymer under oscillatory shear are presented, and conclusions drawn.

\section{Structure formation}

The development of order in LC BCPs must occur subject to constraints imposed by both the liquid crystalline mesophase and the block copolymer microdomain morphology. Thus the structure formed may in some cases represent a compromise between the structures preferred by the two separate ordering tendencies. The equilibrium structure presented by a liquid crystalline block copolymer may be understood by giving consideration to the critical issues listed below.

Relative positions of thermal transition temperatures. Preferred anchoring condition of mesogens at IMDS. Volume fraction of block constituted by mesogen.

Curvature of IMDS.

Size of microdomain relative to size of mesophase.

Strength and density of LC defects.

Symmetry of native mesophase and that of the emergent microphase separated structure.

Coupling of microdomains and LC mesophase to external fields.

The relative location of thermal transitions and the preferred anchoring condition of the mesogens at the IMDS are particularly important, as these characteristics influence the path dependence and the packing of the mesogens into their confining volumes, respectively.

\subsection{Influence of thermal character}

The thermal transitions of interest in LC BCPs are the block copolymer order to disorder transition at $T_{\mathrm{ODT}}$, the LC mesophase structural transitions such as at $T_{\mathrm{Sm}(\mathrm{A})-\mathrm{N}}, T_{\mathrm{N}-\mathrm{I}}$ and $T_{\mathrm{Sm} \text { (A)-I }}$ and the glass transitions of the LC and coil blocks, at $T_{\mathrm{g}}^{\mathrm{A}}$ and $T_{\mathrm{g}}^{\mathrm{B}}$. Typically, for samples of reasonable molecular weight, because of the large chemical incompatibility between the blocks due to the presence of the mesogen, $T_{\mathrm{ODT}}$ for LC BCPs is quite high and thus inaccessible with- out thermal degradation of the material. In systems with very low molecular weight, $T_{\mathrm{ODT}}$ may actually be accessible, and one can witness microphase separation driven expressly by the formation of LC mesophases on passage through $T_{\mathrm{I}-}$ LC [7]. In this case, the morphology which forms ultimately will depend on whether the microphase separation that accompanies liquid crystallization can direct the LC mesophases to create periodically spaced microdomains. In crystallizable block copolymers, for instance, it is usually the case that crystallization induced phase separation results in the production of a spherulitic-lamellar superstructure which gives rise to a disordered microdomain structure [30]. In the case of liquid crystalline diblock copolymers, structure formation from the isotropic melt above $T_{\mathrm{g}}^{\mathrm{A}}$ and $T_{\mathrm{g}}^{\mathrm{B}}$ as well as $T_{\text {I-LC }}$ may proceed by two routes, following microphase separation on passage through the ODT. If $T_{\text {I-LC }}>T_{\mathrm{g}}^{\mathrm{B}}$ then liquid crystallinity develops in a rubbery host block (A) which is tethered to another rubbery block (B) which then vitrifies. If $T_{\mathrm{I}-\mathrm{LC}}<T_{\mathrm{g}}^{\mathrm{B}}$ then mesophase formation is less able to affect the final morphology as it occurs within or around glassy microdomains.

The isothermal evaporation of solvent from initially isotropic solutions may result in the formation of different morphologies depending, of course, on the solvent used. The solvent may affect the final morphology by either encouraging or suppressing the formation of a lyotropic mesophase before microphase separation, which would occur on passage through an $O D C$ or order-disorder concentration. Additionally, preferential swelling of one phase by the solvent may skew the volume fraction of the blocks, resulting in the formation of an initial microdomain structure that would not be arrived at by cooling from an isotropic melt where the volume fractions are uninfluenced by a preferential solvent.

\subsection{Influence of boundary anchoring conditions}

Boundary conditions at the IMDS play a crucial role in determining the structure of the mesophase in the LC BCP, and may also preclude the formation of some microdomain or LC structures entirely. Typically, if unrestrained, the mesogens of the LC block will have a preferred orientation to the IMDS. This anchoring condition may be homogeneous, homeotropic, or involve the mesogen lying at some angle to the IMDS. The effect of the anchoring condition is evaluated with attention paid to the size of the microdomains relative to that of the preferred mesophase, the preferred curvature of the IMDS, the volume fraction of the mesogen in the LC block, and the strength of the anchoring in the preferred orientation. The size and form of the microdomains determine the curvature of the IMDS. A very strong preferred anchoring condition could destabilize and thus eliminate microdomain structures in which satisfaction of the IMDS boundary condition for the mesophase would result in the production of significant defect energy (a high enough number and density of defects produced by the 

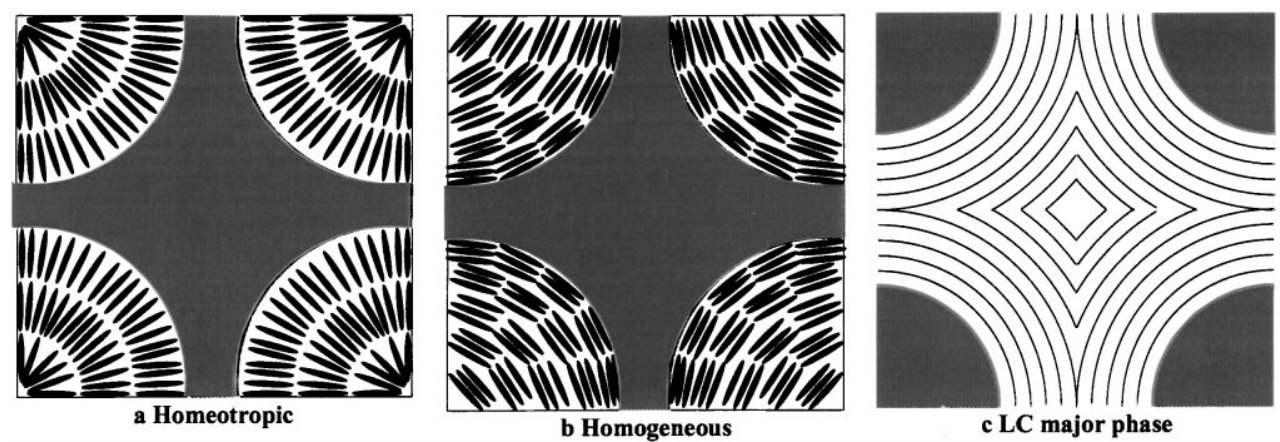

Fig. 3. Packing frustration of mesogens in the smectic A mesophase confined to block copolymer microdomains. Figures are cross-sections of an array of quarter-spheres for mesogens in the minor ((a) and (b)) and major (c) domains. The mesogen spacing is $5 \AA$, layer period is $\approx 30 \AA$, the radius of curvature of the microdomains is $100 \AA$ in each case, and the separation between the sphere centers is $500 \AA$. The lines shown in $\mathbf{c}$ are the layer lines for homeotropic boundary conditions and the director field for homogeneous boundary conditions. The two are related by $90^{\circ}$. Note that figure $\mathbf{c}$ is on a larger scale than $\mathbf{a}$ and $\mathbf{b}$. Point defects of strength $s=+1$ exist at the centers of the spheres in $\mathbf{a}$ and $\mathbf{b}$ and a line defect of $s=-1$ resides at the center of the array of spheres in $\mathbf{c}$.

preferred anchoring). Strong evidence for this effect is given by the reversible structural transition from spheres to cylinders on passage through $T_{\mathrm{I}-\mathrm{N}}$ reported by Gronski and coworkers [31]. Alternatively, the mesophase may change, such that some other, perhaps even less ordered, mesophase is produced, but the boundary condition is satisfied and a stable microdomain structure is realized, as suggested by the data of Fischer et al. [12] which shows a change of mesophase from smectic A in lamellar microdomains to nematic in spherical ones. If the volume fraction of the mesogens in the LC block is small, then the preference of anchoring condition of the mesogen takes on a smaller role in determining the final structure of the LC BCP because the physical effect of the IMDS may be screened from the mesogens by the backbone coil portion of the LC host block. If the anchoring condition is weak, then it may change to suit efficient packing of the mesogens into a stable mesophase within the microdomain.

The formation of smectic mesophases within spheres would not be expected due to the high curvature of the IMDS which excludes efficient mesogen packing in parallel layers. If the spheres were very large relative to the thick- ness of the smectic layers, however, the packing frustration would be reduced, and smectic mesophases may indeed be observed. The proximity of the IMDS presented by microphase separated block copolymers makes defects in a mesophase confined by the IMDS highly unstable, depending on the anchoring condition-the degeneracy of planar anchoring at the IMDS makes it possible for defects to exist in-plane within the microdomain, but not out-of-plane. Direct competition occurs between satisfying the anchoring condition and the elimination of defects due to packing which is prescribed by the IMDS anchoring, for a given mesophase structure. Examples of packing frustrations for a smectic A mesophase confined to block copolymer domains are illustrated in Fig. 3.

The enthalpic penalty for the formation of defects in the matrix is less than that for their formation in the minority phase since the gradients in order parameter are more severe in the confined domains than in the larger matrix phase. Where the anchoring condition and the inherent LC and microdomain symmetries make for efficient packing in a confining microdomain, one can expect very low levels of

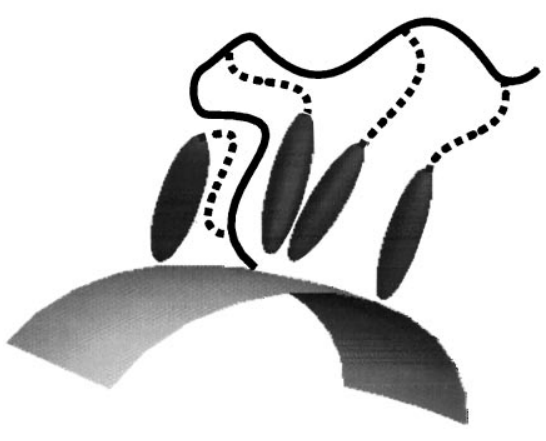

a Long spacer

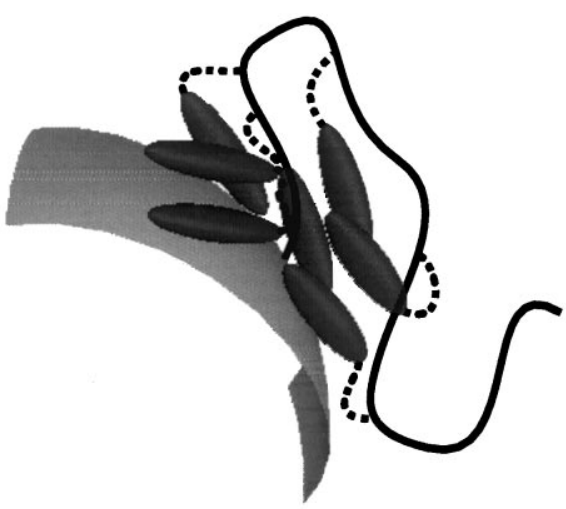

b Short spacer

Fig. 4. Influence of long and short spacers on mesogen anchoring at IMDS. (a) Homeotropic anchoring is possible given a relatively long spacer whereas (b) a shorter spacer necessitates homogeneous anchoring. 
defects within the confined mesophase. This is indeed supported by the data discussed later.

From geometric considerations alone, for mesogens which are decoupled from the polymer backbone by relatively short spacers, homeotropic anchoring may be inaccessible. Conversely, mesogens with long spacers are better able to adopt the homeotropic boundary condition. The situation is illustrated schematically in Fig. 4. Recently, some evidence along these lines has emerged [16,17]. Currently, the effect of spacer length on mesogen anchoring is being investigated in hydrogen bonded analogs of these side group LC BCPs by our group [32].

\subsection{Predicting morphology}

The energetic penalties associated with packing mesogens into the smectic A mesophase in a restricted geometry can be assessed by considering the free energies associated with deformation of this mesophase, and the anchoring of the mesogens at the IMDS in their preferred or easy orientation. In liquid crystalline block copolymers, the confining microdomains are on the order of $0.05 \mu \mathrm{m}$ in scale. Thus we can describe the ordering of the mesophase using Landaude Gennes theory.

Confinement of smectic mesophases into volumes with curved surfaces may result in the deformation of the mesophase, depending on the anchoring conditions, as mentioned above. Such spatial variation in the local order parameter of the mesophase causes elastic strains to develop within the mesophase. For smectic layers of thickness $d$ parallel to the director $\mathbf{n}$, there is a spatial density modulation $\rho(\mathbf{r})=$ $\rho_{0}\left\{1+\operatorname{Re}\left[\psi(\mathbf{r}) \mathrm{e}^{\mathrm{i} q z}\right]\right\}$ where $\psi(\mathbf{r})$ is the complex smectic order parameter and $q=2 \pi / d$. The complex density order parameter, $\psi(\mathbf{r})=\eta(\mathbf{r}) \mathrm{e}^{\mathrm{i} \phi(\mathbf{r})}$, where the positional order parameter $\eta(\mathbf{r})$ describes the degree of layer ordering and $\phi(\mathbf{r})$ is a phase factor defining the position of the layers. Landau-de Gennes theory [33,34] gives the free energy density written in Eq. (1a).

$$
\begin{aligned}
f(\psi, \mathbf{n})= & \frac{1}{2} A|\psi|^{2}+\frac{1}{4} C|\psi|^{4}+c_{\text {par }}|(\nabla \cdot \mathbf{n}-\mathrm{i} q) \psi|^{2} \\
& +c_{\text {perp }}|(\mathbf{n} \times \nabla) \psi|^{2}+\frac{1}{2}\left[K_{11}(\nabla \cdot \mathbf{n})^{2}\right. \\
& \left.+K_{22}(\mathbf{n} \cdot \nabla \times \mathbf{n})^{2}+K_{33}(\mathbf{n} \times \nabla \times \mathbf{n})^{2}\right] \\
& +\left[-\frac{1}{2} K_{24} \nabla[\mathbf{n}(\nabla \cdot \mathbf{n})+\mathbf{n} \times \nabla \times \mathbf{n}]+K_{13} \nabla[\mathbf{n}(\nabla \cdot \mathbf{n})]\right]
\end{aligned}
$$

$f_{\mathrm{e}}^{\mathrm{b}}=\frac{1}{2} K_{11}(\nabla \times \mathbf{n})^{2}+\frac{1}{2} B\left(\frac{\partial u}{\partial z}\right)^{2}$

The first two terms in $f(\psi, \mathbf{n})$ describe mean field contributions, i.e. they are expansions in terms of the order parameter for the free energy of the uniform mesophase that is a function of temperature only. The remaining terms depend on gradients in, and thus the spatial derivatives of the local order parameter and account for the contribution to the free energy due to deformation of the mesophase. $A$ and $C$ are coefficients of the Landau-de Gennes expansion, $c_{\mathrm{par}}$ is the smectic compressibility constant, related to the de Gennes compressibility $B, c_{\text {perp }}$ is the bend constant associated with tilting the director away from the layer normal, and $K_{11}, K_{22}$, $K_{33}, K_{24}, K_{13}$, are the Frank nematic elasticity coefficients for director splay, twist, bend, saddle-splay and mixed splaybend deformation. $f_{\mathrm{e}}^{\mathrm{b}}$ defined in Eq. (1b) is the free energy density associated strictly with the deformation of a bulk smectic A mesophase. $(\partial u / \partial z)^{2}$ gives the curvature of the layers of spacing $u$ with respect to the local layer normal $z$. The surface energy contribution to the free energy results from deviations from ideal anchoring conditions. Thus it is a function of the difference between the actual surface order parameter and the "easy" or ideal surface order parameter. It may be explicitly expressed in the manner of Rapini and Papoular [35] in terms of anchoring angles or the corresponding vector products as shown in Eq. (2).

$$
\begin{aligned}
& f_{\mathrm{S}}\left(Q_{i j}^{\mathrm{S}}\right)=f_{\mathrm{S}}\left(Q_{i j}^{\mathrm{e}}\right)+\frac{1}{2} \tilde{W}\left(Q_{i j}^{\mathrm{S}}-Q_{i j}^{\mathrm{e}}\right)^{2} \\
& f_{\mathrm{S}}=f_{\mathrm{S}}\left(Q_{i j}^{\mathrm{e}}\right)+\frac{1}{2}\left(W_{\theta} \cos ^{2} \phi+W_{\phi} \sin ^{2} \phi\right) \sin ^{2} \theta \\
& F_{\mathrm{S}}=-\frac{1}{2} W_{\theta} \int_{\Sigma}(\mathbf{n} \cdot \hat{\mathbf{z}})^{2} \mathrm{~d}^{2} \mathbf{r}+\frac{1}{2} W_{\phi} \int_{\Sigma}(\mathbf{n} \cdot \hat{\mathbf{x}})^{2} \mathrm{~d}^{2} \mathbf{r}
\end{aligned}
$$

where $Q_{i j}^{\mathrm{S}}$ is the actual surface order parameter and $Q_{i j}^{\mathrm{e}}$ is the "easy" surface order parameter which is obtained by minimizing $f_{\mathrm{S}}$. Here $\mathbf{z}$ is the easy axis in the surface plane and $\mathbf{x}$ is the direction perpendicular to the surface plane. $W_{\theta}$ is the polar anchoring energy associated with out-of-plane director variations from the easy direction and $W_{\phi}$ is the azimuthal anchoring energy associated with in-plane director deviations from the easy direction, as defined in Eq. (3) relative to the surface tension, $\gamma_{\mathrm{s}}$. For degenerate planar anchoring, $W_{\theta} \rightarrow 0$ and $W_{\phi} \rightarrow \infty$.

$W_{\theta}=\frac{1}{2}\left(\frac{\partial^{2} \gamma_{\mathrm{s}}}{\partial \theta^{2}}\right)_{\phi}\left(\theta_{\mathrm{s}}, \phi_{\mathrm{s}}\right) \quad W_{\phi}=\frac{1}{2}\left(\frac{\partial^{2} \gamma_{\mathrm{s}}}{\partial \phi^{2}}\right)_{\theta}\left(\theta_{\mathrm{s}}, \phi_{\mathrm{s}}\right)$

The director field that exists within a confined mesophase is that which minimizes the excess free energy of the system, which consists, in the absence of external fields, of the elastic strain and anchoring energy terms. Using the expressions for the anchoring energy density [35,36] and the elastic strain energy provided in Eqs. (1) and (2), the free energy functional $G[\mathbf{n}(\mathbf{r})]$ is defined in Eq. (4).

Finding the equilibrium director field of the confined mesophase involves solving a variational problem that seeks the minimum of this free energy, subject to appropriate boundary conditions.

$G[\mathbf{n}(\mathbf{r})]=\int_{V} f(\psi, \mathbf{n}) \mathrm{d} V+\int_{\Sigma} f_{\mathrm{S}}(\mathbf{n}) \mathrm{d} \Sigma$

The coupling of the liquid crystal mesophase energetics to those of the block copolymer is through the size and form of 
Table 1

Summary of results obtained for various compositions of $\mathrm{P}(\mathrm{S}-b$-ILC) ( ${ }^{*}$ DSC and SAXS indicate microphase separation but the sample lacked long range order and could not be successfully imaged by TEM)

\begin{tabular}{|c|c|c|c|c|}
\hline $\begin{array}{l}M_{\mathrm{n}}[\mathrm{PS} / \mathrm{PI}-\mathrm{LC}] \\
(\mathrm{kg} / \mathrm{mol})\end{array}$ & $M_{\mathrm{w}} / M_{\mathrm{n}}$ & $\Phi_{\mathrm{PI}-\mathrm{LC}}$ & $\begin{array}{l}T_{\mathrm{Sm}(\mathrm{A})-\mathrm{I}} \\
\left({ }^{\circ} \mathrm{C}\right)\end{array}$ & Morphology \\
\hline $215 / 36$ & 1.09 & 0.13 & 118 & Disordered $^{*}$ \\
\hline $107 / 29$ & 1.07 & 0.20 & 189 & Cylinders of LC \\
\hline $176 / 55$ & 1.13 & 0.22 & 173 & Cylinders of LC \\
\hline $176 / 78$ & 1.15 & 0.29 & 165 & Cylinders of LC \\
\hline $66 / 60$ & 1.12 & 0.45 & 153 & Lamellae \\
\hline $8 / 8$ & 1.09 & 0.48 & 135 & Lamellae \\
\hline $59 / 62$ & 1.10 & 0.49 & 158 & Lamellae \\
\hline $27 / 53$ & 1.13 & 0.64 & 165 & Cylinders of PS \\
\hline $13 / 43$ & 1.10 & 0.75 & 187 & Cylinders of PS \\
\hline $14 / 72$ & 1.24 & 0.82 & 190 & Cylinders of PS \\
\hline $18 / 180$ & 1.30 & 0.91 & 169 & Cylinders of PS \\
\hline 78 & 1.15 & 1.00 & 171 & Homopolymer \\
\hline
\end{tabular}

the block copolymer microdomains which determine their volume and the IMDS surface area, $V$ and $\Sigma$. The chemical incompatibility between the backbones of the two blocks is generally enhanced by the presence of the mesogen, resulting in highly stretched chains which increase the size of the microdomains above that which one would expect from non-LC functionalized diblocks of similar molecular weight [37]. Even at temperatures above $T_{\mathrm{I}-\mathrm{LC}}$, there is not a significant change in microdomain spacing which suggests that the large microdomain sizes are due more to the chemical effect of strong block segregation rather than purely a physical effect of liquid crystalline mesophase formation [20]. Any additional chain stretching that further decreases the surface to volume ratio of the mesophase and the curvature of the IMDS, thus aiding defect relaxation in the mesophase, comes with a decrease in conformational entropy of the block copolymer backbone but also a decrease in the amount of excess free energy due to the presence of the IMDS of the block copolymer. The global free energy of the system must take these contributions into account along with those for the liquid crystal mesophase. Neglecting kinetic effects, the equilibrium morphology of the system is simply that which minimizes this global free energy. Whether the anchoring condition that is observed is the thermodynamically preferred orientation that the mesogens would have at a bulk surface or is guaranteed only by the fact that the block backbones are stretched due to strong segregation, or both, is not known.

\section{Experimental results and discussion}

A well defined series of diblock copolymers consisting of a monodisperse styrene segment paired with an isoprene which was functionalized with an azobenzene based mesogen and an $n$-pentane spacer has been studied by us for a number of years. The compositions ranged from $\Phi_{\mathrm{PI}-\mathrm{LC}}=$ 0.13 to 1.0 . The homopolymer LC formed a smectic A mesophase with a layer spacing of $31 \AA$ and an average

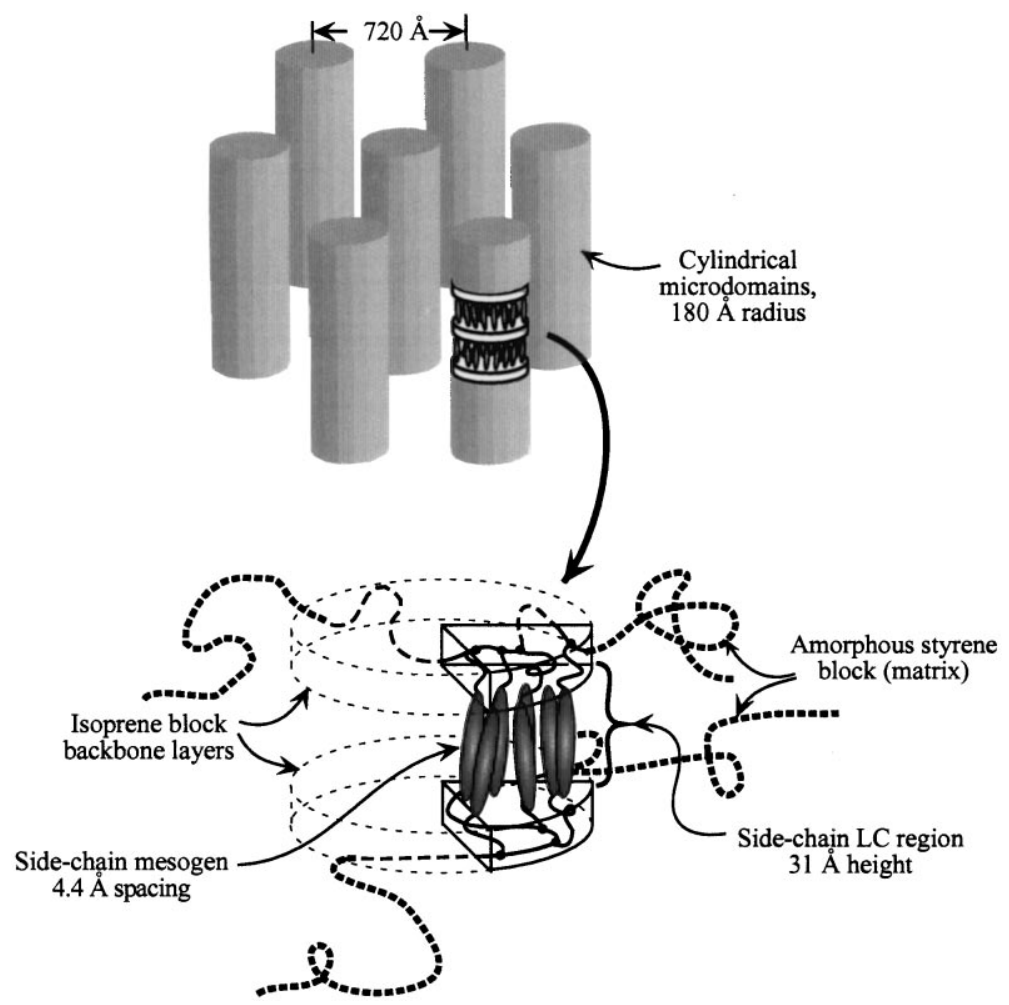

Fig. 5. Homogeneous boundary condition for smectic A mesophases confined to cylinder microdomains. 

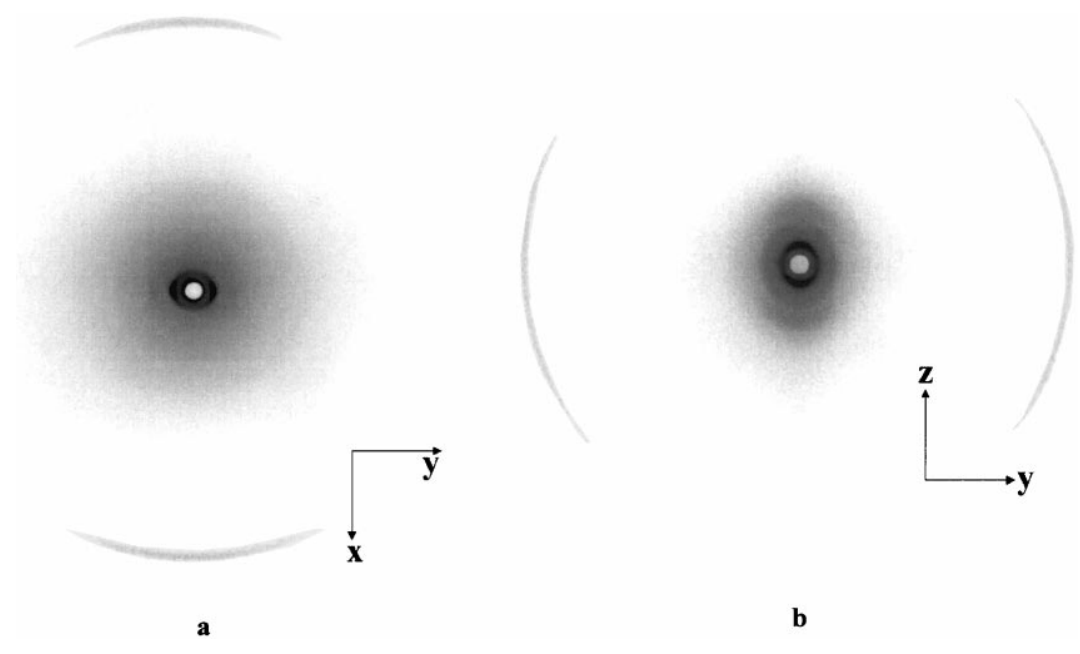

Fig. 6. Ex situ scattering results. (a) $\mathrm{P}\left(\mathrm{S}-b\right.$-ILC) $27 / 53$ sheared at $180^{\circ} \mathrm{C}-\mathrm{X}$-ray beam directed along $\mathbf{z}$, parallel to the velocity gradient. Flow is along $\mathbf{x}$. Scattering from the $d_{1010}$ and $d_{1120}$ reflections of the hexagonal lattice of the PS cylinders is enhanced along the $y$-direction indicating the cylinder axes have aligned with the flow direction (parallel alignment). The $\mathrm{d}_{001}$ smectic layer reflection appears only after cooling and implies a preferred homogeneous anchoring of the mesogens with respect to the IMDS. (b) $\mathrm{P}\left(\mathrm{S}-b\right.$-ILC) $27 / 53$ sheared at $150^{\circ} \mathrm{C}$. X-rays incident parallel to the flow direction, $\mathbf{x}$. Scattering from the $\mathrm{d}_{1010}$ reflection of the PS cylinders is now enhanced along the $\mathbf{z}$ direction indicating that the cylinder axes are aligning along the vorticity direction of the flow (transverse alignment). Simultaneously, the $\mathrm{d}_{001}$ reflection of the smectic layers concentrates along the $y$ direction indicating that the LC layers take on a perpendicular alignment. This arrangement also allows the preferred homogeneous anchoring of the mesogens with respect to the IMDS during flow alignment in the LC regime (see structural model 8d).

inter-mesogen packing distance of around $4.4 \AA$. The homopolymer smectic A to isotropic transition temperature was $171^{\circ} \mathrm{C}$.

The compositions of the azobenzene series and the morphologies determined by TEM and SAXS for each sample are given in Table 1. Samples were prepared by slowly evaporating a $5 \mathrm{wt}$.\% solution of the polymer in toluene followed by thermal annealing at $140^{\circ} \mathrm{C}$ in vacuum. Both small and wide angle $\mathrm{X}$-ray scattering were used to determine the orientation of the mesogens with respect to the IMDS in globally well oriented samples produced by roll-casting [29] and oscillatory shear. For cylinders of the LC block, as well as cylinders of styrene and lamellae, the mesogens were found to orient parallel to the IMDS (i.e. homogeneous boundary condition), forming smectic A mesophases. Fig. 5 illustrates the efficient packing of mesogens in smectic A layers within a cylinder.

The presence of PS cylinders at a volume fraction more typically associated with spheres for coil-coil block copolymers, $\mathrm{P}\left(\mathrm{S}-b\right.$-ILC) $18 / 180, \Phi_{\mathrm{PI}-\mathrm{LC}}=0.91$, may be due to the high enthalpic penalties that would be associated with packing smectic layers around spheres. The high volume fraction of the mesogens in the isoprene block of these polymers undoubtedly lends significance to the role of the mesogen's preferred IMDS anchoring condition in determining structure. The results of an in situ SAXS/WAXS (wide-angle Xray scattering) study of the oscillatory shear induced orientation of PS cylinders in a PI-LC matrix reveal this clearly, and are discussed below.

\subsection{Oscillatory shear orientation}

The LC homopolymer was subjected to oscillatory shear and found to adopt a parallel orientation of the layers with respect to the plane of shear. The conditions used were $\epsilon=$ $100 \%, \nu=0.5 \mathrm{~Hz}, 165^{\circ} \mathrm{C}$. P(S-b-ILC) $27 / 53$ was also subjected to oscillatory shear using the same frequency and amplitude, and the orientation of the PS cylinders was found to vary depending on the presence or absence of smectic layers in the matrix [38]. As shown in the ex situ SAXS data of Fig. 6, taken from [38], at $T>T_{\mathrm{Sm}(\mathrm{A})-\mathrm{I}}$, the cylinders oriented parallel to the shear flow, whereas at $T<$ $T_{\mathrm{Sm}(\mathrm{A})-\mathrm{I}}$, they were found to adopt a transverse orientation, in which their long axes are aligned along the neutral axis, and the LC layers a perpendicular orientation with the layer normal along $\mathbf{y}$. Data taken in situ showed that upon thermal isotropization of the LC matrix during shearing, the cylinders re-oriented from the transverse into the parallel orientation, which is the commonly observed orientation in coilcoil diblock copolymers. Upon cooling, in the absence of shear, the smectic layers formed with their normals oriented parallel to the shear direction, $\mathbf{x}$. This is the same mesogenIMDS boundary arrangement as in the transverse orientation. The transverse-perpendicular (Fig. 7d) orientation represents a compromise between the parallel cylinder and parallel smectic layers structures, which are preferred by the microphase separated structure and the LC mesophase individually. This compromise is forced by the strength of the homogeneous anchoring condition - a violation of it would have made the simultaneous parallel orientations of the microstructure and smectic layers possible (Fig. 7c). The 


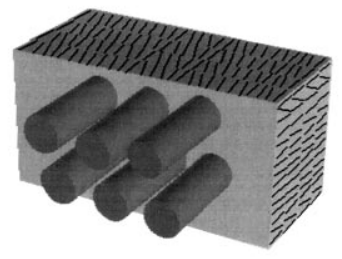

a

PARALLEL-TRANSVERSE

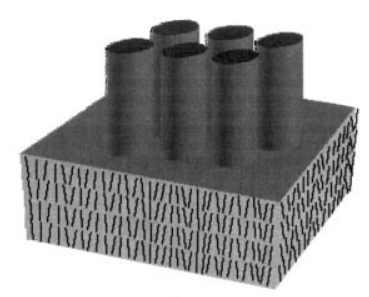

b

PERPENDICULAR-PARALLEL<smiles>[Y]C([Y])[V]</smiles>

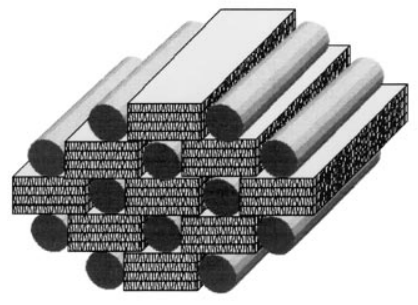

PARALLEL-PARALLEL

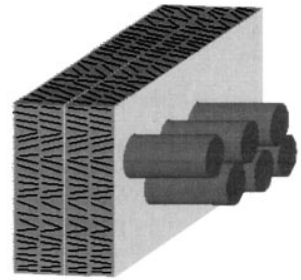

d

TRANSVERSE-PERPENDICULAR

Fig. 7. Schematic structural models of smectic layers and cylindrical microdomains with two types of boundary conditions for the mesogens with respect to the IMDS. Flow is along $\mathbf{x}$ and vorticity along $\mathbf{z}$. (a) Parallel-transverse: model which places the cylinders in their preferred parallel orientation along the flow direction and with homogeneous boundary conditions for the mesogens. The smectic layers are in the unfavorable transverse orientation. (b) Perpendicularparallel: model which places the smectic layers in their preferred parallel orientation with respect to the shear flow with homogeneous boundary conditions for the mesogens. The cylinders are in the unfavorable perpendicular orientation. (c) Parallel-parallel: model which places both the cylinders and the smectic layers in their preferred parallel orientations but the homogeneous boundary condition for the mesogens is lost. (d) Transverse-perpendicular: model which provides a compromise structure in which the cylinders are transverse and the layers are perpendicular but which maintains homogeneous boundary conditions of the mesogens. This is the actual structure of $\mathrm{P}\left(\mathrm{S}-b\right.$-ILC) $27 / 53$ after shearing at $150^{\circ} \mathrm{C}$.

same experiment performed with cylinders of the LC in a PS matrix, P(S-b-ILC) $176 / 55$, gave the same results, but the time scale for the adoption of the transverse orientation was significantly longer, this indicative of the lower volume fraction of LC in the material as a whole. Fig. 7 illustrates schematically four possible candidate morphologies under shear, as well as the observed transverse-perpendicular morphology which represents the compromise between the antagonistic orientation tendencies present in the material.

\subsection{Influence of confinement: reduction of defects}

Confinement of the mesophase within small diameter cylindrical microdomains tends to eliminate defects due to the high surface to volume ratio of the microdomains, otherwise expressed in terms of the average proximity of a mesogen within the microdomain to the IMDS surrounding it.
The azimuthal spread of the X-rays scattered by the smectic layers and by the microphase separated cylinders provides two useful estimates of the levels of hierarchical order within the material. The angular distribution of X-rays due to scattering from the smectic layers results from the superposition of spread due to any non-uniform smectic layer orientation within the microdomains as well as that due to the non-uniform microdomain orientation. For P(S- $b$-ILC) $176 / 55$, oscillatory shear at $190^{\circ} \mathrm{C}, \epsilon=150 \%, \nu=1 \mathrm{~Hz}$ for 60 min was used to produce highly oriented cylinders within which smectic layers with high correlation of orientation between layers is observed, as demonstrated by the data in Fig. 8a. An order parameter, the second component of the harmonic orientation distribution function, of $S=0.93$ for the cylinders and $S=0.91$ for the smectic layers within the cylinders is calculated in the manner of Windle [39].

The low concentration of defects present in mesophases confined to cylinders versus matrix mesophases or 


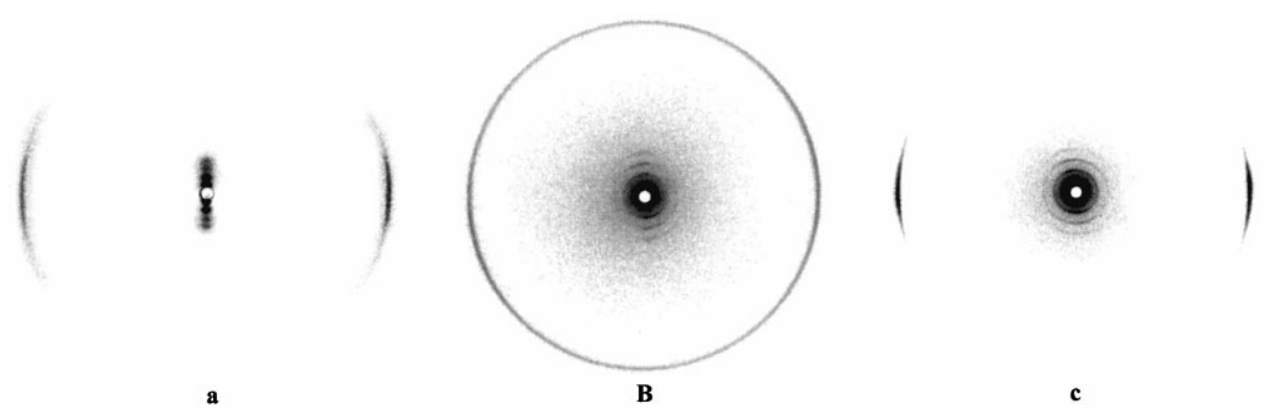

Fig. 8. SAXS of oriented samples taken at room temperature. X-rays are incident perpendicular to the plane of shear. Shear axis is horizontal to the page. (a) Highly ordered LC mesophases produced after cooling inside cylindrical microdomains oriented above $T_{\mathrm{I}-\mathrm{LC} .} S_{\mathrm{CYL}}=0.93, S_{\mathrm{Sm}(\mathrm{A})}=0.91$ (b) Predominantly parallel lamellae oriented above $T_{\mathrm{I}-\mathrm{LC}}$. The isotropic ring is due to the disordered smectic layers which form on cooling. (c) Predominantly parallel lamellae oriented below $T_{\mathrm{I}-\mathrm{LC}}$ with oriented smectic layers within.

mesophases within a lamellar microdomain structure is manifested by the optical clarity of samples in which the microstructures have been oriented either above or below the clearing temperature of the mesophase. For sample compositions in which the equilibrium microstructures are LC cylinders, as well as PS cylinders and PS/LC lamellae, oscillatory shear above $T_{\mathrm{I}-\mathrm{LC}}$ produces films that are highly transparent at the shearing temperature. However, on cooling below $T_{\mathrm{I}-\mathrm{LC}}$, this transparency is lost for the LC matrix/ PS cylinder and LC/PS lamellar samples, but retained for the LC cylinder sample. The loss of transparency is due to the scattering of light by defects which form within the LC matrix or lamellae. In addition, in the lamellar sample, the boundary condition is degenerate-smectic layers may lie in any plane parallel to the surface of the lamellae and still satisfy the homogeneous anchoring condition at the IMDS. As a consequence, there will be domain boundaries between regions with different director orientation, within one block copolymer microdomain. As expected, shearing below $T_{\text {I-LC }}$ produces transparent films for the matrix or cylinders of LC, and lamellae, since most defects within the LC are removed by the shearing process. Subsequent heating above the clearing transition of the mesophase followed by cooling below the clearing temperature again resulted in a loss of optical clarity only for LC matrix and LC lamellar-for these samples, defects spawned readily on cooling into the LC phase, whereas repeated heating above the clearing temperature of the LC mesophases for samples in which the LC was confined to cylindrical microdomains produced no losses in optical clarity upon cooling into the LC regime. As shown in Fig. 8b, an isotropic scattering ring is found at room temperature for the smectic layers in the lamellar sample which is oriented above the clearing temperature of the LC, whereas for samples oriented below $T_{\mathrm{I}-\mathrm{LC}}$ (Fig. $8 c)$ the scattered intensity due to the smectic layers is concentrated into sharp arcs perpendicular to the lamellae, consistent with planar anchoring of the mesogens at the IMDS.

\subsection{Influence of confinement: thermal characteristics}

The confinement of liquid crystals is known to have effects on their thermal behavior [40]. DSC was used to characterize the thermal properties of the materials. Transition temperatures were taken using second heating scans with heating rates of $10^{\circ} \mathrm{C} / \mathrm{s}$. The results are presented graphically in Fig. 9. The clearing entropy was found to be a function of the composition of the copolymer, increasing
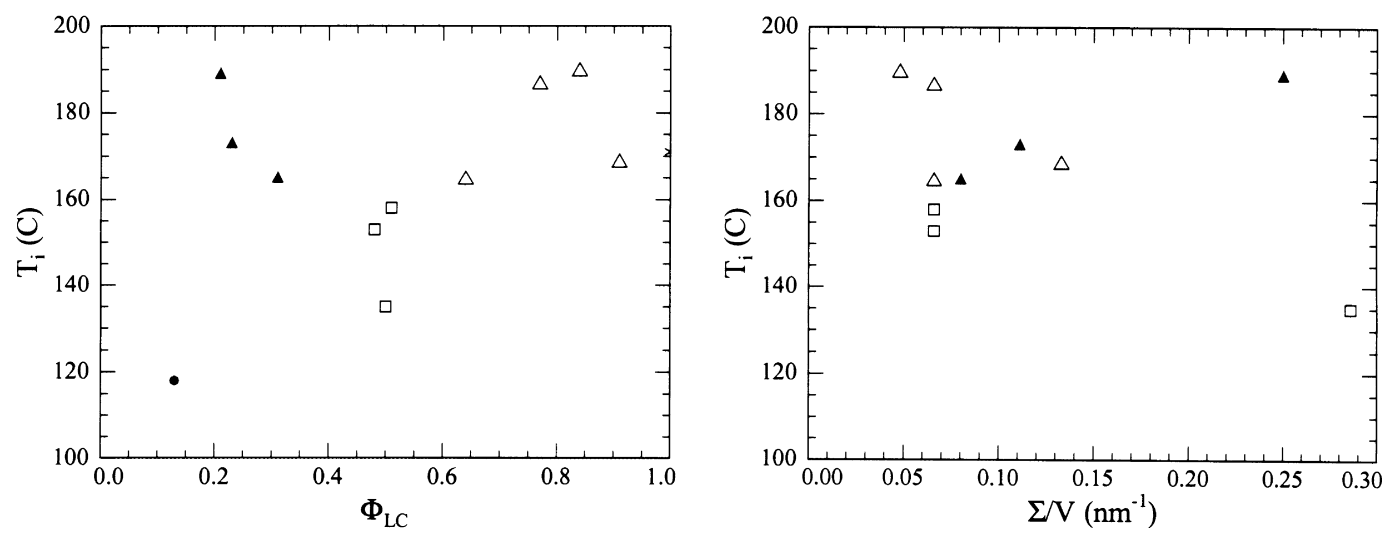

Fig. 9. Summary of $T_{\mathrm{I}-\mathrm{LC}}$ data for P(S-b-ILC) series. $\Phi_{\mathrm{PI}-\mathrm{LC}}=0.13: \bullet$; PS cylinders: $\triangle$; PILC cylinders: $\mathbf{\Delta}$; lamellae: $\square$; homopolymer: $*$. surface to volume ratios of the LC phase $\Sigma / V$ calculated on the basis of domain sizes and volume fractions. 
with increasing $\Phi_{\mathrm{PI}-\mathrm{LC}}$. For samples in which the average LC molar mass is in the vicinity of $65,000 \mathrm{~g} / \mathrm{mol}$., there was an increase in the clearing enthalpy and entropy in moving from cylinders of LC to lamellae and finally to the LC matrix samples. However, this trend was not reflected in the transition temperatures of the samples. Typically, the influence of phase size or surface proximity on a firstorder transition may be gauged using the Gibbs-Thompson relation written in Eq. (5), where $T^{0}$ is the transition temperature for the bulk material, $\Delta H$ is the heat of fusion per unit mass, $\sigma$ is the mean surface free energy, $V$ is the specific volume and $l$ is a structural parameter characterizing the smallest dimension of the phase.

It seems reasonable that the increase in the clearing temperature of the mesophases in cylinder forming block copolymers is due to the different principal curvatures $(K)$ of the curved IMDS $\left(K_{\text {radial }}=1 / R_{\text {cyl }} ; K_{\text {axial }}=0\right)$, and thus lack of degeneracy in the mesogen anchoring condition, which stabilizes the mesophase by lowering the surface energy term, $\sigma$, in Eq. (5). The influence of molecular weight on clearing temperature can be dispensed with somewhat by considering the effect of surface to volume ratio on the clearing temperature as graphed in Fig. 9. Clearly there is delineation in $T_{\mathrm{I}-\mathrm{LC}}$ based on IMDS curvature, i.e. between the lamellae and cylinder forming samples (both PS and PI-LC cylinders), as mentioned above. Similar results have been obtained by Yamada et al. [41] using a poly(styrene- $b$-methacrylate) block copolymer, in which a cyano-biphenyl mesogen is attached to the methacrylate block via a six carbon spacer. Here, they also found homogeneous mesogen anchoring at the IMDS and a $15-20^{\circ} \mathrm{C}$ difference between the higher clearing temperature of their LC cylinder sample vs. the lower temperatures of the lamellar samples.

$T(l, \sigma)=T^{0}\left[1-\frac{V \sigma}{l \Delta H}\right]$

It is speculated that the decrease in clearing entropy relative to the homopolymer LC is due to confinement of the mesogens to microdomains. The presence of a pseudo-isotropic layer at the IMDS may account for some of this effect, but this should not be expected given the high enthalpic penalty associated with the existence of such a layer within a small confined mesophase. It is more probable that such an isotropic layer may exist in samples in which the matrix is formed by the LC block, and the minority phase forms domains with surfaces of high curvature in more than one direction, e.g. spheres. In such a sample, the shape of the IMDS would not permit satisfaction of the preferred mesogen anchoring condition. Since the extent of the matrix is large, an isotropic IMDS layer would be more readily accommodated within the LC mesophase. Micro-area electron diffraction from ultra-thin sections of a bulk oriented sample could be used to probe this, though Gronski has already concluded, on the basis of ${ }^{2} \mathrm{H} \mathrm{NMR}$, that such isotropic layers do not in fact exist [11].
Finally, from consideration of the three samples that formed LC cylinders, we see that there is an increase in the clearing temperature with decreasing $\mathrm{LC}$ volume fraction, and a corresponding increase in clearing temperature as a function of surface to volume ratio. Though no strong conclusion can be drawn from only three data points, the trend supports our supposition that the inter-material dividing surface plays a critical role in the thermal stabilization of this series of materials. Typically, increasing LC molecular weight in homopolymers will increase clearing temperatures up to some limit, but we see here that even the increase in LC molecular weight cannot offset the decrease in clearing temperature due to the decrease in the surface to volume ratio of the larger diameter cylindrical LC microdomains.

\section{Summary and outlook}

Many factors come into play in the determination of the final structure of side group LC block copolymers. The persistence of the PS cylinder phase to unusually low volume fractions points to a strong influence of the LC phase in determining the microdomain structure. Further, the LC phase has a distinct effect on the orientation of the block microdomains during oscillatory shear. Mesogens of the LC phase were found to orient strictly parallel to the IMDS of the microdomains, though it is not known whether this is a thermodynamic effect, a function of backbone stretching coupled with spacer length, or both. The presence of curvature in the IMDS clearly lends thermal stability to the LC mesophase whether it exists as the confined phase or as the matrix phase. In addition, confined LC mesophases were found to be of very high order due to the average proximity of the IMDS to the confined mesogens.

The creation of functional electro-optic or piezo-electric devices using side group LC block copolymers relies on careful control of the microstructure to optimize a desired response of the material. In particular, the application of these block copolymers as display materials is being pursued. The orientation of the microstructure in the thin films used in display devices is important in determining the overall properties of the device. Epitaxy between a matrix mesophase and highly oriented PTFE monolayers has been successfully used to orient these P(S- $b$-ILC) block copolymers [32]. Such methods of orientation, along with electric and magnetic field alignment will probably be preferred for producing well oriented films for various electro-optic applications. Hydrogen bonded LC diblock copolymers are currently being studied for similar potential applications [32]. Interestingly, it has recently been found possible to generate microphase separated structures by careful complexation of less than stoichiometric amounts of an appropriate mesogen with a suitable polymer backbone [32]. Similar morphological characterization of these new materials as presented here for the covalently bonded $\mathrm{P}(\mathrm{S}-b-$ ILC) block copolymers is underway. 


\section{Acknowledgements}

This work was supported by the NSF under grant DMR 97-05271 and made use of MRSEC shared facilities of the MIT CMSE supported by NSF under DMR 94-00334. The authors wish to acknowledge the staff of the National Synchrotron Light Source at Brookhaven National Lab including Dr Fengji Yeh and Prof Benjamin Hsiao. We also thank Prof Murugappan Muthukumar (UMassAmherst) and Prof Paula Hammond (MIT) for very helpful discussions.

\section{References}

[1] Quiram DJ, Register RA, Marchand GR, Ryan AJ. Macromolecules 1997;30:8338-43.

[2] Mao GP, Ober CK. Acta Polym 1997;48:405-22.

[3] Muthukumar M, Ober CK, Thomas EL. Science 1997;277:1225-32.

[4] Öge T, Zentel R. Macromol Chem Phys 1996;197:1805-13.

[5] Zentel R, Brehmer M. Chemtech 1995(May):41-8.

[6] Mao GP, Wang J, Ober CK, O’Rourke MJ, Thomas EL, Brehmer M, Zentel R. Chem Mater 1998;10:1538-45.

[7] Zheng WY, Hammond PT. Macromolecules 1997;31:711-21.

[8] Brehmer M, Mao GP, Ober CK, Zentel R. Ferroelectric block copolymers. In: Zentel R, Galli G, Ober CK, editors. Macromolecular Symposia number 117, May 1997. p. 175-9.

[9] Pugh C, Percec V. Polym Preprints 1986;27:366-7.

[10] Adams J, Gronski W. Makromol Chem Rapid Commun 1989;10:553-7.

[11] Tefehne MA, Gronski W. Macromol Rapid Commun 1996;17:30511.

[12] Fischer H, Poser S, Arnold M, Frank W. Macromolecules 1994;27:7133-8.

[13] Fischer H, Poser S, Arnold M. Liq Cryst 1994;8:503-9.

[14] Fischer H, Poser S, Arnold M. Macromolecules 1995;28:6957-62.

[15] Mao GP, Wang J, Clingman SR, Ober CK, Chen JT, Thomas EL. Macromolecules 1997;30:2556-67.

[16] Anthamatten M, Zheng WY, Hammond PT. Macromolecules 1999;32:4838-48.

[17] Anthamatten M, Hammond PT. Macromolecules 1999;32:8066-76.

[18] Moment A, Miranda R, Hammond PT. Macromol Rapid Commun 1998;19:573-9.
[19] Mao GP. Molecular design, synthesis, characterization and processing of liquid crystalline diblock copolymers. PhD thesis, Cornell University, 1997.

[20] Chen JT. Influence of an LC block on microstructure and optical properties of block copolymers. PhD thesis, Massachusetts Institute of Technology, 1997.

[21] Chen JT, Thomas EL, Ober CK, Hwang SS. Macromolecules 1995;28:1688-97.

[22] Chen JT, Thomas EL, Ober CK, Mao GP. Science 1996;273:343-6.

[23] Osuji C, Chen JT, Mao GP, Ober CK, Thomas EL. Structure development in side group liquid crystalline diblock copolymers. In: Morishima Y, Norisuye T, Tashiro K, editors. Molecular interactions and time-space organization in macromolecular systems, Berlin: Springer, 1999. p. 9-28.

[24] Ober CK, Mao GP, Wang J, Kramer EJ, Chen JT, Thomas EL. Multiple length scale self-organization in liquid crystalline block copolymers. In Zentel R, Galli G, Ober CK, editors. Macromolecular Symposia number 117, May 1997. p. 141-52.

[25] Wang JG, Mao GP, Ober CK, Kramer EJ. Macromolecules 1997;30:1906-14.

[26] Keller A, Pedemonte E, Willmouth FM. Nature 1970;225:538.

[27] Mathis A, Hadziioannou G, Skoulios A. Polym Engng Sci 1977; $17: 570$.

[28] Amundson K, Helfand E, Davis DD, Quan X, Patel SS, Smith SD. Macromolecules 1991;24:6546-8.

[29] Albalak R, Thomas EL. J Polym Sci (Phys) 1993;31:37-46.

[30] Rangarajan P, Register RA, Fetters LJ, Bras W, Naylor S, Ryan AJ. Macromolecules 1995;28:4932-8.

[31] Sanger J, Gronski W, Maas S, Stuhn B, Heck B. Macromolecules 1997;30:6783-7.

[32] Osuji C, Chao CY, Ober CK, Thomas EL. In preparation.

[33] de Gennes PG, Prost J. The physics of liquid crystals. 2nd ed. Oxford: Clarendon Press, 1993.

[34] Linhananta A, Sullivan DE. Phys Rev A 1991;44:8189-97.

[35] Rapini A, Papoular M. J Physique Coll 1969;30:C4-54.

[36] Nobili M, Durand G. Phys Rev A 1992;46:R6174-7.

[37] Walther M, Finkelmann H. Prog Polym Sci 1996;21:951-79.

[38] Osuji C, Zhang Y, Mao GP, Ober CK, Thomas EL. Macromolecules 1999;32:7703-6.

[39] Mitchell GR, Windle AH. Developments in crystalline polymers, vol 2. Amsterdam: Elsevier, 1988 (chap 3).

[40] Crawford GP, Zumer S. Liquid crystals in complex geometries formed by polymer and porous networks. Taylor and Francis, 1996.

[41] Yamada M, Itoh T, Nakagawa R, Hirao A, Nakahama S, Watanabe J. Macromolecules 1999;32:282-9. 